\title{
Correction \\ Correction: King et al. Surface-Rupturing Historical Earthquakes in Australia and Their Environmental Effects: New Insights from Re-Analyses of Observational Data. Geosciences 2019, 9, 408
}

\author{
Tamarah R. King ${ }^{1, * \mathbb{D}}$, Mark Quigley ${ }^{1}$ and Dan Clark ${ }^{2}$ \\ 1 School of Earth Sciences, University of Melbourne, Melbourne 3010, Australia; mark.quigley@unimelb.edu.au \\ 2 Geoscience Australia, Canberra 2609, Australia; dan.clark@ga.gov.au \\ * Correspondence: tamarah.king@unimelb.edu.au
}

check for

updates

Citation: King, T.R.; Quigley, M.; Clark, D. Correction: King et al. Surface-Rupturing Historical Earthquakes in Australia and Their Environmental Effects: New Insights from Re-Analyses of Observational Data. Geosciences 2019, 9, 408.

Geosciences 2022, 12, 81 .

https://doi.org/10.3390/

geosciences12020081

Received: 19 January 2022

Accepted: 21 January 2022

Published: 10 February 2022

Publisher's Note: MDPI stays neutral with regard to jurisdictional claims in published maps and institutional affiliations.

Copyright: () 2022 by the authors. Licensee MDPI, Basel, Switzerland. This article is an open access article distributed under the terms and conditions of the Creative Commons Attribution (CC BY) license (https:// creativecommons.org/licenses/by/ $4.0 /)$.
The authors would like to correct the published article [1]:

Table 1 on page 2 of the article requires three corrections:

1. The published maximum vertical displacement $(\mathrm{m})$ of the Tennant Creek $1(\mathrm{Ku}-$ nayungku) NT earthquake was $0.9 \mathrm{~m}$, not $10.9 \mathrm{~m}$;

2. The date of the Lake Muir, WA surface rupturing earthquake was 16/09/2018, not 08/11/2018 (this was the date of a Mw 5.2 aftershock, see [2] for details);

3. Due to a calculation error described below, the average net-slip $(\mathrm{m})$ calculated from this paper for the Cadoux earthquake should be $0.45 \mathrm{~m}$, not $0.54 \mathrm{~m}$.

The updated and correct Table 1 is:

Table 1. Summary of known historic Australian surface rupturing earthquakes and relevant references.

\begin{tabular}{|c|c|c|c|c|c|c|c|c|c|}
\hline \multirow[b]{2}{*}{ Name } & \multirow[b]{2}{*}{ Fig. } & \multirow[b]{2}{*}{$\begin{array}{l}\text { Magnitude } \\
\text { (Mw) [11] }\end{array}$} & \multirow[b]{2}{*}{ Date (UTC) } & \multicolumn{3}{|c|}{ This Paper: } & \multicolumn{2}{|c|}{ Published } & \multirow[b]{2}{*}{$\begin{array}{c}\text { Relevant } \\
\text { References }\end{array}$} \\
\hline & & & & $\begin{array}{l}\text { Length } \\
(\mathrm{km})\end{array}$ & Dip & $\begin{array}{l}\text { Avg. } \\
\text { Net-slip } \\
\text { (m) }\end{array}$ & $\begin{array}{l}\text { Length } \\
(\mathrm{km})\end{array}$ & $\begin{array}{l}\text { Max. Vert. } \\
\text { Disp. (m) }\end{array}$ & \\
\hline Meckering, WA & 1 & 6.59 & $14 / 10 / 1968$ & $40 \pm 5$ & $35^{\circ} \pm 10$ & 1.78 & 37 & 2.5 & {$[12-37]$} \\
\hline Calingiri, WA & 8 & 5.03 & $10 / 03 / 1970$ & $3.3 \pm 0.2$ & $20^{\circ} \pm 10$ & 0.46 & 3.3 & 0.4 & $\begin{array}{c}{[23-} \\
25,38,39]\end{array}$ \\
\hline $\begin{array}{c}\text { Cadoux, WA } \\
\text { Marryat Creek, SA }\end{array}$ & $\begin{array}{l}4 \\
5\end{array}$ & $\begin{array}{l}6.1 \\
5.7\end{array}$ & $\begin{array}{l}02 / 06 / 1979 \\
30 / 03 / 1986\end{array}$ & $\begin{array}{l}20 \pm 5 \\
13 \pm 1\end{array}$ & $\begin{array}{l}60^{\circ} \pm 30 \\
40^{\circ} \pm 10\end{array}$ & $\begin{array}{l}0.45 \\
0.31\end{array}$ & $\begin{array}{l}14 \\
13\end{array}$ & $\begin{array}{l}1.4 \\
0.9\end{array}$ & $\begin{array}{c}{[28,40-45]} \\
{[1,28,46-49]}\end{array}$ \\
\hline $\begin{array}{c}\text { Tennant Creek } 1 \\
\text { (Kunayungku) NT } \\
\text { Tennant Creek } 2\end{array}$ & 7 & 6.27 & $22 / 01 / 1988$ & $9 \pm 1$ & $40^{\circ} \pm 5$ & 0.55 & 10.2 & 0.9 & [1,49-69] \\
\hline $\begin{array}{l}\text { Tennant Creek } 2 \\
\text { (Lake Surprise } \\
\text { west) }\end{array}$ & 6 & 6.44 & $22 / 01 / 1988$ & $9 \pm 2$ & $60^{\circ} \pm 10$ & 0.84 & 6.7 & 1.1 & [1,49-69] \\
\hline $\begin{array}{c}\text { Tennant Creek } 3 \\
\text { (Lake Surprise east) }\end{array}$ & 3 & 6.58 & $22 / 01 / 1988$ & $16 \pm 0.5$ & $35^{\circ} \pm 5$ & 1.23 & 16 & 1.8 & {$[1,49-69]$} \\
\hline $\begin{array}{l}\text { Katanning, WA } \\
\text { Pukatja, SA }\end{array}$ & $\begin{array}{c}10 \\
9\end{array}$ & $\begin{array}{c}4.7 \\
5.18\end{array}$ & $\begin{array}{l}10 / 10 / 2007 \\
23 / 03 / 2012\end{array}$ & $\begin{array}{l}0.5 \pm 0.5 \\
1.3 \pm 0.3\end{array}$ & $\begin{array}{c}40^{\circ} \pm 5 \\
30^{\circ} \pm 10\end{array}$ & $\begin{array}{c}0.2 \\
0.25\end{array}$ & $\begin{array}{l}1.26 \\
1.6\end{array}$ & $\begin{array}{l}0.1 \\
0.5\end{array}$ & $\begin{array}{l}{[70,71]} \\
{[9,72]}\end{array}$ \\
\hline Petermann, NT & 2 & 6.1 & $20 / 05 / 2016$ & $21 \pm 0.5$ & $30^{\circ} \pm 5$ & 0.42 & 20 & 1.0 & [73-78] \\
\hline Lake Muir, WA & & 5.3 & $16 / 09 / 2018$ & & & & 3 & 0.5 & [79] \\
\hline
\end{tabular}

Other literature with relevant analysis or data regarding historic ruptures: [80-98].

Net-slip values calculated for the Cadoux earthquake were found to be incorrect due to an excel error, where the incorrect data cells were referenced in a function calculating net-slip. This changes the net-slip values published in the Supplementary information, the average net-slip value for Cadoux in Table 1 (see above), the net-slip values in Figure $5 \mathrm{~d}$, and the net-slip and derivative values in Tables 6, 9 and 10 .

These errors were identified while working on a subsequent publication [3], which builds on the results in this paper [1]. The Cadoux net-slip values were corrected prior to the analyses in [3] and the results in that paper are unaffected by the corrections outlined here.

The authors apologize for any inconvenience this has caused for the readers. The changes do not affect the scientific results of this paper [1] or of the subsequent work [3]. The manuscript will be updated, and the original version will remain online on the article web page, with a reference to this correction. 
The updated Figure 5d graph is:

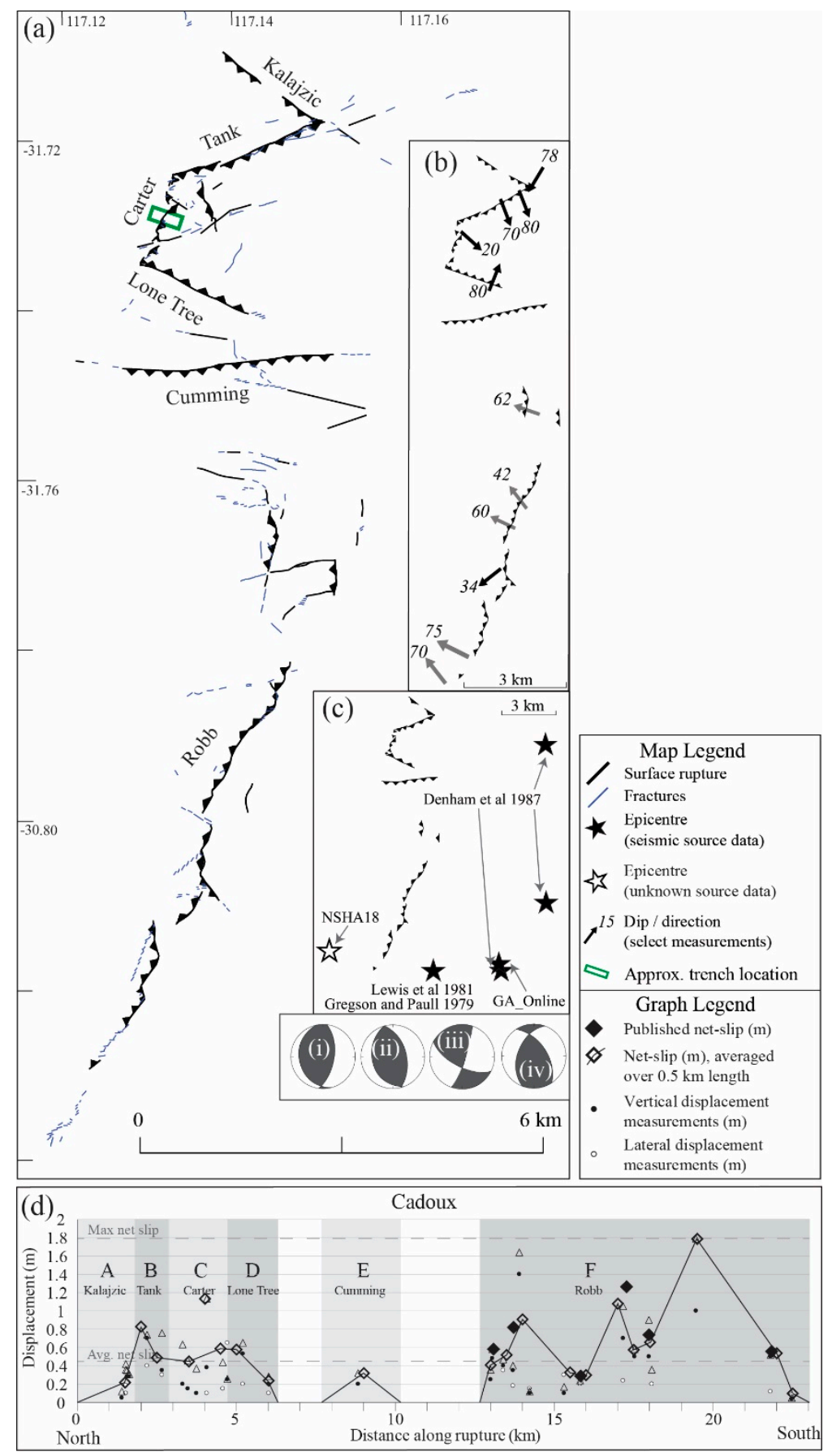

Figure 5. $1979 \mathrm{Mw}$ 6.1 Cadoux earthquake (a) rupture scarps and fracturing involved in the Cadoux rupture with named faults [41], focal mechanisms from (i) Denham et al., 1987 (ii) Fredrich et al., 1988 (iii) Everingham and Smith (unpublished, Lewis et al., 1981) (iv) CMT (b) available dip measurements, black where directly measured and grey were calculated based on available displacement measurements [41] (c) published epicenter locations (d) graph along-rupture of vertical and lateral displacement measurements and calculated net slip [41] and net slip calculated from available data averaged over $0.5 \mathrm{~km}$ increments (this study). 
The previous Figure 5d with incorrect net-slip values was:

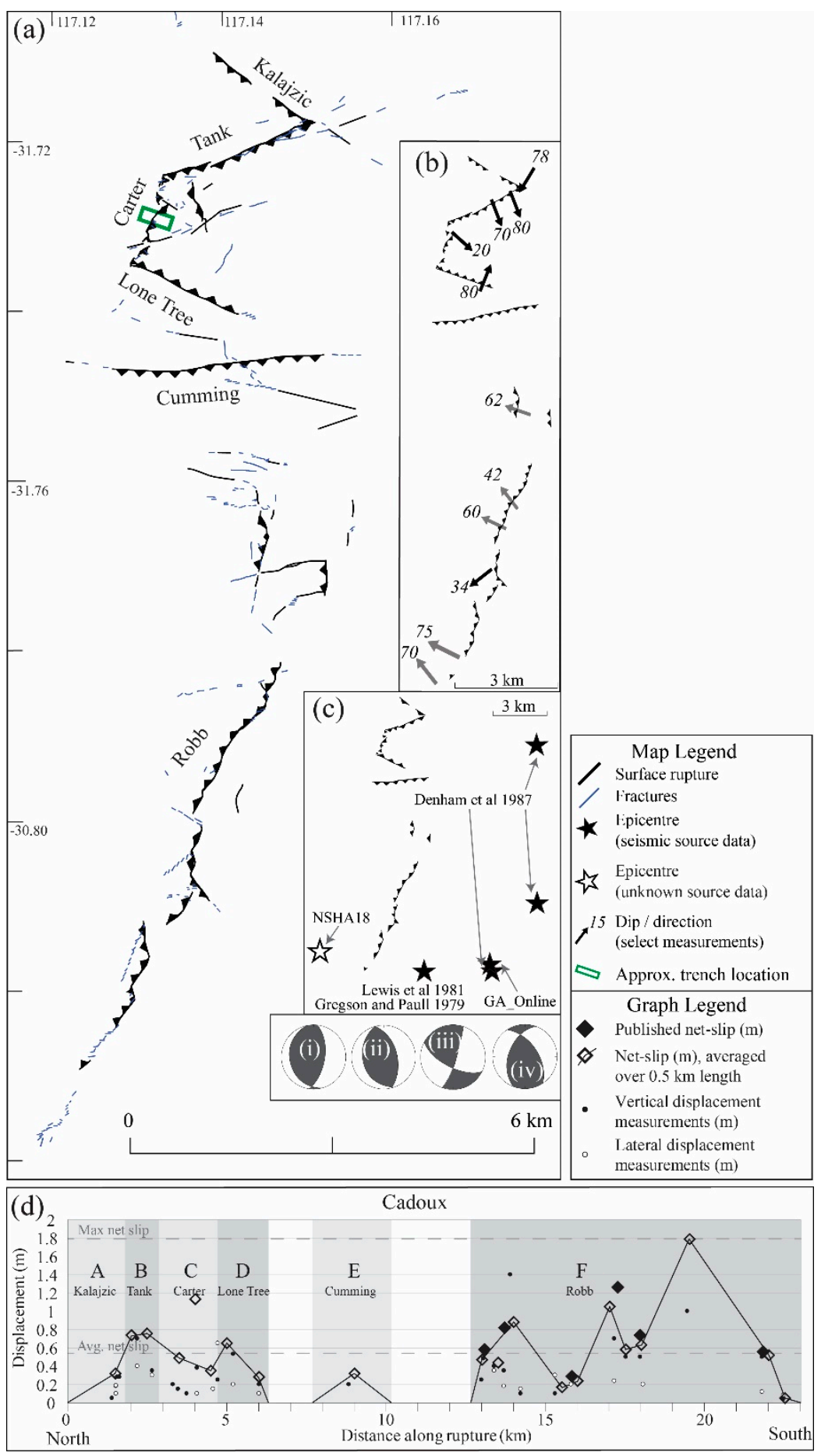

Figure 5. 1979 Mw 6.1 Cadoux earthquake (a) rupture scarps and fracturing involved in the Cadoux rupture with named faults [41], focal mechanisms from (i) Denham et al., 1987 (ii) Fredrich et al., 1988 (iii) Everingham and Smith (unpublished, Lewis et al., 1981) (iv) CMT (b) available dip measurements, black where directly measured and grey were calculated based on available displacement measurements [41] (c) published epicenter locations (d) graph along-rupture of vertical and lateral displacement measurements and calculated net slip [41] and net slip calculated from available data averaged over $0.5 \mathrm{~km}$ increments (this study). 
The corrected net-slip values for the Cadoux earthquake are:

\begin{tabular}{|c|c|c|c|}
\hline Cadoux & 117.1492 & -30.7165 & 0.06 \\
\hline Cadoux & 117.1502 & -30.717 & 0.29 \\
\hline Cadoux & 117.1538 & -30.7186 & 0.15 \\
\hline Cadoux & 117.1549 & -30.7194 & 0.27 \\
\hline Cadoux & 117.1496 & -30.7176 & 0.29 \\
\hline Cadoux & 117.1446 & -30.7198 & 0.83 \\
\hline Cadoux & 117.1403 & -30.722 & 0.49 \\
\hline Cadoux & 117.1342 & -30.7233 & 0.59 \\
\hline Cadoux & 117.1334 & -30.7237 & 0.44 \\
\hline Cadoux & 117.1327 & -30.7262 & 0.3 \\
\hline Cadoux & 117.1327 & -30.7279 & 1.13 \\
\hline Cadoux & 117.1332 & -30.7312 & 0.47 \\
\hline Cadoux & 117.1305 & -30.7329 & 0.71 \\
\hline Cadoux & 117.1324 & -30.735 & 0.58 \\
\hline Cadoux & 117.1395 & -30.7387 & 0.24 \\
\hline Cadoux & 117.1373 & -30.7464 & 0.32 \\
\hline Cadoux & 117.1392 & -30.7591 & 0 \\
\hline Cadoux & 117.1524 & -30.7503 & 0.2 \\
\hline Cadoux & 117.1524 & -30.7534 & 0.02 \\
\hline Cadoux & 117.1392 & -30.7591 & 0.29 \\
\hline Cadoux & 117.1445 & -30.7614 & 0.52 \\
\hline Cadoux & 117.1464 & -30.7638 & 0.59 \\
\hline Cadoux & 117.1454 & -30.7677 & 0.44 \\
\hline Cadoux & 117.1447 & -30.7697 & 1.62 \\
\hline Cadoux & 117.1464 & -30.7704 & 0.2 \\
\hline Cadoux & 117.1511 & -30.773 & 0.33 \\
\hline Cadoux & 117.1468 & -30.7756 & 0.3 \\
\hline Cadoux & 117.144 & -30.7878 & 1.08 \\
\hline Cadoux & 117.1415 & -30.7904 & 0.58 \\
\hline Cadoux & 117.1398 & -30.7943 & 0.9 \\
\hline Cadoux & 117.1423 & -30.7968 & 0.42 \\
\hline Cadoux & 117.1372 & -30.8077 & 1.79 \\
\hline Cadoux & 117.1279 & -30.8239 & 0.54 \\
\hline Cadoux & 117.124 & -30.829 & 0.1 \\
\hline
\end{tabular}

The corrected Table 6 is:

Corrections: Cadoux "Avg. net slip" (0.54 to 0.45) and “\% Diff". (70\% to 75\%)

The corrected Table 9 is:

Correction: Cadoux "Avg. Net-slip (m)" (0.54 to $0.45 \mathrm{~m}$ ) and "Maximum slip rate (m/Myr)" "min." (0.6 to $0.7 \mathrm{~m} / \mathrm{Myr}$ ), "max." (10.6 to $12.7 \mathrm{~m} / \mathrm{Myr})$, "mean" (5.6 to $6.7 \mathrm{~m} / \mathrm{Myr}$ )

The corrected Table 10 is:

Correction: Cadoux "Avg net slip" (0.54 to $0.45 \mathrm{~m}$ ) and "\% diff" (-503.7\% to -624.4\%) 
Table 6. Summary of surface measurements for each rupture.

\begin{tabular}{|c|c|c|c|c|c|c|c|c|c|c|c|c|c|c|c|c|c|c|}
\hline \multirow[b]{2}{*}{ Rupture } & \multirow[b]{2}{*}{ Refs. } & \multirow[b]{2}{*}{ Method $^{3}$} & \multirow[b]{2}{*}{ Shape $^{4}$} & \multicolumn{3}{|c|}{ Published } & \multicolumn{3}{|c|}{ Simplified Faults ${ }^{1}$} & \multicolumn{2}{|c|}{ Preferred: } & \multicolumn{6}{|c|}{ Displacement $^{2}(\mathrm{~m})$} & \multirow[b]{2}{*}{$\begin{array}{c}\text { Disp. } \\
\text { Profile } \\
\text { Shape }^{6}\end{array}$} \\
\hline & & & & $\begin{array}{l}\text { Length } \\
(\mathbf{k m})\end{array}$ & Kin. & $\underset{\text { Range }}{\text { Dip }}$ & $\mathrm{N}=$ & $\begin{array}{c}\text { Sum } \\
\text { Length } \\
(\mathbf{k m})\end{array}$ & $\begin{array}{l}\text { \% Diff. } \\
\text { Publ. }\end{array}$ & $\begin{array}{l}\text { Length } \\
(\mathrm{km})\end{array}$ & Dip & $\begin{array}{l}\text { Max } \\
\text { Vert. } \\
\text { Disp. }\end{array}$ & $\begin{array}{l}\text { Avg. } \\
\text { Vert. } \\
\text { Disp. }\end{array}$ & $\%$ Diff. & $\underset{\text { Slip }}{\text { Max Net }}$ & $\begin{array}{l}\text { Avg. Net } \\
\text { Slip }{ }^{5}\end{array}$ & $\%$ Diff & \\
\hline Meckering & [25] & $\mathrm{FW} ; \mathrm{A} ; \mathrm{S}_{\mathrm{B}}$ & $\mathrm{CC}$ & 37 & $R(D)$ & $15-54^{\circ}$ & 4 & 44.4 & $+20 \%$ & $40 \pm 5$ & $35^{\circ} \pm 10$ & 1.98 & 0.97 & $51 \%$ & 3.7 & 1.78 & $52 \%$ & S. Tg. \\
\hline Calingiri & [25] & FW & $\mathrm{s}$ & 3.3 & $\mathrm{R}(\mathrm{S})$ & $12-31^{\circ}$ & 1 & 3.3 & $0 \%$ & $3.3 \pm 0.2$ & $20^{\circ} \pm 10$ & 0.38 & 0.15 & $61 \%$ & 1.26 & 0.46 & $63 \%$ & AS. Tg. \\
\hline Cadoux & [41] & $\mathrm{FW} ; \mathrm{A} ; \mathrm{S}_{\mathrm{B}}$ & $\mathrm{CC} / \mathrm{S}$ & 14 & $\mathrm{R}$ & $20-80^{\circ}$ & 6 & 20.6 & $+47 \%$ & $20 \pm 5$ & $60^{\circ} \pm 30$ & 1.4 & 0.35 & $75 \%$ & 1.79 & 0.45 & $75 \%$ & AS. Tg. \\
\hline Marryat Creek & {$[48]$} & $\mathrm{FW} ; \mathrm{A} ; \mathrm{S}_{\mathrm{C}}$ & $\mathrm{CC}$ & 13 & $\mathrm{R}$ & $36-60^{\circ}$ & 3 & 13.6 & $+4 \%$ & $13 \pm 1$ & $40^{\circ} \pm 10$ & 0.9 & 0.21 & $77 \%$ & 1.07 & 0.31 & $71 \%$ & Avg. \\
\hline Kunayungku & [63] & $\mathrm{FW} ; \mathrm{A} ; \mathrm{S}_{\mathrm{C}}$ & $\mathrm{s}$ & 10.2 & $\mathrm{R}$ & $58^{\circ}$ & 1 & 8.6 & $-15 \%$ & $9 \pm 1$ & $40^{\circ} \pm 5$ & 0.9 & 0.36 & $60 \%$ & 1.41 & 0.55 & $61 \%$ & S. Tg \\
\hline (LS west foot-wall) & [63] & & & 3.1 & $\mathrm{R}$ & & 1 & 10.1 & $+51 \%$ & $9 \pm 2$ & $60^{\circ} \pm 10$ & 0.74 & 0.43 & $42 \%$ & 1.16 & 0.9 & $22 \%$ & Avg. \\
\hline Lake Surprise east & [63] & $\mathrm{FW} ; \mathrm{A} ; \mathrm{S}_{\mathrm{C}}$ & $\mathrm{CC}$ & 16 & $\mathrm{R}$ & $28-30^{\circ}$ & 2 & 15.3 & $-4 \%$ & $16 \pm 0.5$ & $35^{\circ} \pm 5$ & 1.8 & 0.61 & $66 \%$ & 3.6 & 1.23 & $66 \%$ & Avg \\
\hline Katanning (visible) $^{7}$ & {$[70,71]$} & & $\mathrm{S}$ & 0.3 & $\mathrm{R}$ & & 1 & 0.3 & $0 \%$ & \multirow{2}{*}{$0.5 \pm 0.5$} & \multirow{2}{*}{$40^{\circ} \pm 5$} & 0.1 & & & & & & \\
\hline Katanning (InSAR) & [70] & In. & & $2.5^{8}$ & & & 1 & 2.2 & $-12 \%$ & & & $0.2^{8}$ & 0.1 & $50 \%$ & $0.32 *$ & $0.2 *$ & $38 \%$ & AS. Tg. \\
\hline Pukatja & [9] & FW & CC & 1.6 & $\mathrm{R}$ & $22-28^{\circ}$ & 1 & 1.0 & $-60 \%$ & $1.3 \pm 0.3$ & $30^{\circ} \pm 10$ & 0.48 & 0.12 & $75 \%$ & 0.96 & 0.25 & $74 \%$ & AS. Sine \\
\hline Petermann (visible) & {$[73,77]$} & $\begin{array}{l}\text { FW; } \mathrm{S}_{C} \\
\text { In; D; SI }\end{array}$ & $\mathrm{CV}$ & 20 & $\mathrm{R}$ & $25-36^{\circ}$ & 3 & 21 & $+5 \%$ & \multirow{2}{*}{$21 \pm 0.5$} & \multirow{2}{*}{$30^{\circ} \pm 5$} & 0.96 & 0.2 & & 1.92 & 0.42 & $78 \%$ & Avg. \\
\hline Petermann (InSAR) & [73] & In. & & 21 & & & 2 & 21.5 & $+2 \%$ & & & & & & & & & \\
\hline
\end{tabular}

$\begin{array}{rrrr}21 & 21.5 & +2 \%\end{array}$

Where mapped primary rupture has a gap/step $>1 \mathrm{~km}$ and/or change in strike $>20^{\circ}$ across a length $>1 \mathrm{~km}$ (except where InSAR is available to validate rupture continuing along strike across gaps $>1 \mathrm{~km})$. Lengths of individual faults available in EarthArXiv reports $[120,121,122,123,124,125,126] .{ }^{2}$ Vertical and lateral displacements digitized from original publications Net slip calculated for this study. ${ }^{3}$ Original mapping method: Field work (FW); aerial photographs (A); surveying (levelling, cadastral or GPS) basic $\left(\mathrm{S}_{\mathrm{B}}\right)$, comprehensive $\left(\mathrm{S}_{\mathrm{C}}\right)$; InSAR (In); Drone (D); Satellite (SI). ${ }^{4}$ Concave (CC) relative to hanging-wall, convex (CV) relative to hanging-wall, straight (minor deviations but overall straight shape). ${ }^{5}$ Length weighted average across $0.5 \mathrm{~km}$ increments (where rupture length $>5 \mathrm{~km}$ ) or $0.1 \mathrm{~km}$ increments (where rupture length $<5 \mathrm{~km}$ ). ${ }^{6}$ Profile shape based on Wesnousky (2008) [7] from visual fit (e.g., not best-fit regression curves): symmetrical (S); asymmetrical (AS); triangle (Tg); sine; average line (Avg). ${ }^{7}$ Katanning visible surface rupture was observed, but no field mapping was conducted [70,71]. Original and subsequent publications describe Katanning length based on best-fit InSAR-derived source parameters (1.26 km) [70], rather than length of InSAR trace $(2.5 \mathrm{~km})$. Offset comes from field estimates $(0.1 \mathrm{~m})$ and fault modelling from InSAR data [70]. 
Table 9. Maximum slip rates based on minimum and maximum bedrock erosion rates [109,138] and length-weighted average net-slip values (Table 6).

\begin{tabular}{|c|c|c|c|c|c|c|c|}
\hline \multirow{2}{*}{ Name } & \multirow{2}{*}{ Rate Applied * } & \multirow{2}{*}{ Mw } & \multirow{2}{*}{ Pref. Length $(\mathrm{km})$} & \multirow{2}{*}{ Avg. Net-Slip (m) } & \multicolumn{3}{|c|}{ Maximum Slip Rate (m/Myr) } \\
\hline & & & & & Min. & Max. & Mean \\
\hline Meckering & $\mathrm{CB}$ & 6.59 & $40 \pm 5$ & 1.78 & 0.2 & 3.2 & 1.7 \\
\hline Calingiri & $\mathrm{CB}$ & $\begin{array}{l}0.09 \\
5.03\end{array}$ & $\begin{array}{l}40 \pm 0.2 \\
3.3 \pm 0.2\end{array}$ & 0.46 & 0.7 & $\begin{array}{ll}0.2 \\
12.4\end{array}$ & 6.6 \\
\hline Cadoux & $\mathrm{CB}$ & 6.1 & $20 \pm 5$ & 0.45 & 0.7 & 12.7 & 6.7 \\
\hline Marryat Creek & $\mathrm{CB}$ & 5.7 & $13 \pm 1$ & 0.31 & 1 & 18.4 & 9.7 \\
\hline Kunayungku & $\mathrm{Q}$ & 6.27 & $9 \pm 1$ & 0.55 & 9.1 & $\begin{array}{l}10.4 \\
18.2\end{array}$ & 13.7 \\
\hline Lake Surprise west & $\mathrm{Q}$ & 6.44 & $9 \pm 2$ & 0.84 & 6 & 11.9 & 8.9 \\
\hline Lake Surprise east* & $\mathrm{Q}$ & $\begin{array}{l}6.47 \\
6.58\end{array}$ & $16 \pm 0.5$ & 1.23 & 4.1 & 8.1 & 6.1 \\
\hline Katanning (InSAR) & $\mathrm{CB}$ & 4.7 & $0.5 \pm 0.5$ & 0.2 & 1.5 & 28.5 & 15 \\
\hline Pukatja & $\mathrm{CB}$ & 5.18 & $1.3 \pm 0.3$ & 0.25 & 1.2 & 22.8 & 12 \\
\hline Petermann & $\mathrm{CB}$ & 6.1 & $21 \pm 0.5$ & 0.42 & 0.7 & 13.6 & 7.2 \\
\hline
\end{tabular}

${ }^{*}$ Erosion rate for crystalline basement $(\mathrm{CB})[138]$; erosion rate for quartzite $(\mathrm{Q})[109]$.

Table 10. Comparisons between calculated magnitude, area and displacement from previous length scaling relationship [148] using surface rupture length from [148] and length from this paper.

\begin{tabular}{|c|c|c|c|c|c|c|c|c|c|c|c|c|c|c|c|c|}
\hline \multirow{2}{*}{ Name } & \multicolumn{2}{|c|}{ Leonard $2014[148]$} & \multicolumn{3}{|c|}{ Calculated [148] } & \multirow{2}{*}{\begin{tabular}{|c|}
$\begin{array}{l}\text { This } \\
\text { Paper }\end{array}$ \\
L (km)
\end{tabular}} & \multicolumn{3}{|c|}{ Calculated (This Paper) } & \multicolumn{3}{|c|}{ Percent Difference Calculated } & \multirow{2}{*}{$\begin{array}{l}\text { Avg. Net } \\
\text { Slip } 5\end{array}$} & \multirow{2}{*}{$\%$ Diff 6} & \multirow{2}{*}{ Mw [11] } & \multirow{2}{*}{$\%$ Diff 6} \\
\hline & $\begin{array}{l}\mathrm{SRL}^{1}{ }^{1} \\
(\mathrm{~km})\end{array}$ & Source & $\mathrm{Mw}^{2}$ & $\underset{\left(\mathrm{km}^{2}\right)}{\mathrm{A}^{3}}$ & $D^{4}(m)$ & & $\mathrm{Mw}^{2}$ & $\underset{\left(\mathbf{k m}^{2}\right)}{\mathrm{A}^{3}}$ & $D^{4}(m)$ & $\mathrm{Mw}$ & $A\left(\mathrm{~km}^{2}\right)$ & $\mathrm{D}(\mathrm{m})$ & & & & \\
\hline Meckering & 37 & [160] & 6.93 & 5546 & 5.44 & 40 & 6.99 & 6316 & 5.8 & $0.9 \%$ & $12.2 \%$ & $6.3 \%$ & 1.78 & $-225.8 \%$ & 6.59 & $-6.1 \%$ \\
\hline Cadoux & 14 & [160] & 6.23 & 1098 & 2.42 & 20 & 6.49 & 1989 & 3.26 & $4.0 \%$ & $44.8 \%$ & $25.8 \%$ & 0.45 & $-624.4 \%$ & 6.1 & $-6.4 \%$ \\
\hline Marryat Creek & 13 & [160] & 6.18 & 970 & 2.27 & 13 & 6.18 & 970 & 2.27 & $0.0 \%$ & $0.0 \%$ & $-0.2 \%$ & 0.31 & $-632.3 \%$ & 5.7 & $-8.4 \%$ \\
\hline Kunayungku & 10.2 & [160] & 6 & 648 & 1.86 & 9 & 5.91 & 526 & 1.67 & $-1.5 \%$ & $-23.2 \%$ & $-11.3 \%$ & 0.55 & $-203.6 \%$ & 6.27 & $5.7 \%$ \\
\hline Lake Surprise west & 6.7 & [160] & 5.7 & 321 & 1.31 & 9 & 5.91 & 526 & 1.67 & $3.6 \%$ & $39.0 \%$ & $21.7 \%$ & 0.84 & $-98.8 \%$ & 6.44 & $8.2 \%$ \\
\hline Katanning (InSAR) & 1.26 & [70] & 4.49 & 20 & 0.33 & 0.5 & 3.82 & 4 & 0.15 & $-17.5 \%$ & $-400.0 \%$ & $-117.6 \%$ & 0.2 & $25.0 \%$ & 4.7 & $18.7 \%$ \\
\hline Pukatja & 1.6 & [9] & 4.66 & 30 & 0.40 & 1.3 & 4.51 & 21 & 0.33 & $-3.3 \%$ & $-42.9 \%$ & $-21.2 \%$ & 0.25 & $-32.0 \%$ & 5.18 & $12.9 \%$ \\
\hline
\end{tabular}

Surface rupture length. ${ }^{2}$ [148]: $\mathrm{M}_{\mathrm{W}}=\mathrm{a}+\mathrm{b}^{*} \log (\mathrm{L}) .{ }^{3}$ [148]: $\mathrm{A}=\mathrm{C}_{1} \mathrm{~L}^{1+\beta} \cdot{ }^{4}[148]$ : $\mathrm{D}=\mathrm{C}_{2} \mathrm{~A}^{1 / 2} \cdot{ }^{5}$ Average net slip calculated in this paper (Table 6). ${ }^{6}$ Percent difference between calculated average displacement and $\mathrm{M}_{\mathrm{w}}$ using length of this paper [148], and average net slip calculated in Table 6, and $\mathrm{M}_{\mathrm{w}}$ of [11]. 


\section{References}

1. King, T.R.; Quigley, M.; Clark, D. Surface-rupturing historical earthquakes in Australia and their environmental effects: New insights from re-analyses of observational data. Geosciences 2019, 9, 408. [CrossRef]

2. Clark, D.; Brennand, S.; Brenn, G.; Garthwaite, M.C.; Dimech, J.; Allen, T.I.; Standen, S. Surface deformation relating to the 2018 Lake Muir earthquake sequence, southwest Western Australia: New insight into stable continental region earthquakes. Solid Earth 2020, 11, 691-717. [CrossRef]

3. Haibin, Y.; Quigley, M.; King, T.R. Surface slip distributions and geometric complexity of intraplate reverse-faulting earthquakes. GSA Bull. 2021, 133, 1909-1929. 